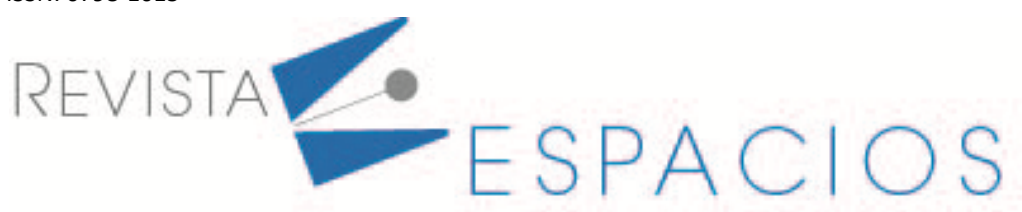

\title{
Correlación entre resultados contables y generación de valor económico de un grupo empresarial colombiano
}

\section{Correlation between accounting results and economic value generation of a colombian business group}

\author{
CANTILLO, Julio C. ${ }^{1}$ \\ DE LA HOZ, Aminta I. ${ }^{2}$ \\ SANTAMARÍA, Álvaro E. ${ }^{3}$
}

\begin{abstract}
Resumen
El objetivo de este trabajo consistió en determinar la existencia de relación entre el valor económico agregado, el valor agregado del mercado, y dos de los principales indicadores de resultados contables: rentabilidad neta y rentabilidad operativa, tomando como referencia los estados financieros (2015 a 2019) de uno de los grupos empresariales más importantes de Colombia. Se concluye que existe una correlación, significativa entre las medidas mencionadas, exaltándose a la información contable en la determinación de valor en las organizaciones.

Palabras clave: contabilidad, valoración, desempeño, rentabilidad.
\end{abstract}

\begin{abstract}
The objective of this work is to determine the existence of the relationship between economic added value, market added value, and two of the main indicators of accounting results: net profitability and operating profitability, taking as reference the financial statements (2015 to 2019) of one of the most important business groups in Colombia. It is concluded that there is a significant correlation between the specific measures, exalting the accounting information in determining the value in organizations. key words: accounting, valuation, performance, profitability.
\end{abstract}

\section{Introducción}

Bajo el modelo económico actual las medidas de desempeño tienen un papel preponderante en la toma de decisiones, éstas proporcionan información valiosa para monitorear la gestión y en especial los avances estratégicos de las organizaciones y los grupos empresariales. En este sentido, las ciencias contables han suministrado información valiosa para la medición del desempeño con enfoque financiero. Estas medidas se han implementado durante las últimas décadas, según Villegas (2016), la expansión de los mercados financieros, con

\footnotetext{
${ }^{1}$ Docente. Facultad de Ciencias Económicas y Administrativas. Universidad de Sucre. Email: jccpsincel@gmail.com

${ }^{2}$ Docente. Facultad de Ciencias Económicas y Administrativas. Universidad de Sucre. Email: amihoz@hotmail.com

${ }^{3}$ Profesor Asociado. Facultad de Ciencias Económicas y Administrativas. Universidad de Sucre. Email: alvaro.santamaria@unisucre.edu.co
} 
un enfoque basado en la maximización del valor para el accionista como doctrina central de gestión, ha generado el surgimiento de medidas enfocadas en la generación de valor económico y financiero.

Las medidas de desempeño financiero basadas en principios de reconocimiento, medición, revelación y presentación contable, según Chari (2009) y Machado \& Da Cuhna (2011) tradicionalmente han sido "insuficientes para la toma de decisiones", en razón a que presentan una serie de limitaciones, dentro de las que se pueden mencionar: información muy agregada y resumida, información con poca capacidad predictiva para establecer y explicar rendimientos futuros, y un diseño con enfoque histórico de los hechos económicos. Es importante señalar lo complejo que es establecer una medida de desempeño financiero adecuado para cada tipo organización o grupo económico, que permita determinar el valor y que sea de amplia aceptación. A partir de estos limitantes y de las exigencias de la nueva doctrina de gestión, desde la literatura se han propuesto una serie de métodos que puedan cumplir con el objetivo de medir el valor económico y financiero de los grupos económicos, dentro de los métodos ampliamente utilizados se destacan el economic valué added (EVA) y el market added valué (VMA).

El economic valué added (EVA), conocido por sus siglas en español como valor económico agregado, es una "medida desarrollada e incorporada a la realidad empresarial por Joel Stern y G. Bennett Stewart III, fundadores de la empresa estadounidense de consultoría empresarial Stern Stewart \& Co" (Li Bonilla, 1969; Machado \& Da Cuhna, 2011; Stewart, 1991). Para Banerjee (2000) "el EVA es una versión modificada de la teoría del valor de los accionistas que busca establecer el beneficio económico a partir de los costos generados por los pasivos y el patrimonio" (p.23). Por su parte, el market added valué (VMA), conocido por sus siglas en inglés como valor agregado del mercado, indica la ganancia generada por los accionistas a partir del valor del mercado de la organización y de los aportes de capital.

Bajo esta mirada y teniendo en cuenta las pocas investigaciones en Colombia de correlación de variables de medición de desempeño financiero se propone analizar ¿Cuál es la relación estadística entre los métodos de medición el Valor Económico Agregado, el valor Agregado del Mercado y los indicadores financieros de resultados contables? Para ello, se analiza los estados financieros correspondientes a los periodos comprendidos entre los años 2015 y 2019 de uno de los principales grupos empresariales colombiano, para el análisis se toma como referencia el modelo planteado por (Machado \& Da Cuhna, 2011).

\subsection{Contextualización teórica}

Las medidas de desempeño enfocadas en la generación de valor son un eje fundamental en el modelo económico actual, el cual tiene como base los principios de acumulación y de maximización de la riqueza de los accionistas. A partir de este hecho, muchos autores han discutido los méritos de las medidas de rendimiento financiero, en especial el valor de mercado añadido (MVA) y el valor añadido económico (EVA). Según Eswara \& Prasad (2015) el MVA consiste en evaluar el efecto de las acciones gerenciales y la acumulación de la riqueza de los accionistas desde la constitución o inicio de una organización, mientras que el EVA evalúa la eficacia gerencial en un periodo determinado (un año); a diferencia del MVA, el EVA no se centra directamente en los valores del mercado y, por lo tanto, se puede aplicar tanto a las organizaciones propiedad del inversor como a las organizaciones sin fines de lucro.

Sin embargo, es importante establecer ¿Por qué el EVA y el MVA son tan populares? La respuesta se encuentra en las múltiples investigaciones que avalan las bondades de estos modelos. En una investigación se afirma que las medidas tradicionales de desempeño, entiéndase las medidas contables, son insuficientes para determinar este tipo de hechos económicos. Los autores concluyen que la aplicación de EVA puede mejorar el rendimiento corporativo. Sin embargo, establecen la idea de que obtener rendimientos que excedan todos los costos de la inversión (incluido el costo del capital invertido) no es un planteamiento nuevo (Brabazon \& Sweeny, 1998). 
Por su parte, Zafiris \& Bayldon (1999) reafirman la solidez esencial de los modelos EVA y MVA, plantean que son conveniente para examinar la viabilidad organizacional, en razón a que aplican un conjunto mixto de datos, es decir, utilizan datos internos de la organización representados en los estados financieros (datos contables) y datos del mercado bursátil. Para estos autores estos dos modelos representan un avance sobre la contabilidad convencional que puede ser resumida en el reconocimiento adecuado de los costos de oportunidad de todo el capital invertido en una empresa, eliminando así el peligro de mal uso y desperdicio de recursos de capital. Enfatizando lo planteado por Farzad, Degel, \& Degner (2020) y Wet \& Hall (2004) en el sentido que el EVA promueve los intereses de los accionistas por su enfoque de creación de riqueza para los accionistas y enfatiza en la mejora continua de valor.

De igual forma, Reddy, Rajesh, \& Reddy (2011) y Stephens \& Bartunek (1997) comparan el EVA con las medidas tradicionales y concluyen que el EVA permite establecer cifras exactas de cuánto va a recibir el accionista al final del ejercicio contable y opinó que el EVA es la medida más adecuada para medir el valor de los accionistas. Este método, según los autores, plantea como principio fundamental que las tasas de interés de financiación de la operación están estrechamente relacionadas con la generación de valor que el valor de mercado de la organización.

Recientemente, Bhasin (2013), estudió la aplicación del EVA junto con otras medidas convencionales en cinco empresas líderes durante un período de cinco años utilizando el análisis de tendencia, regresión y ANOVA. EI autor da crédito a la afirmación de Stewart de que el EVA es superior a otras medidas convencionales no podía probarse más allá de la duda. Pero opina que EVA está ganando popularidad y está siendo utilizado por las empresas como una herramienta de gestión para la gobernanza interna.

Por su parte Eswara \& Prasad (2015), examinaron siete empresas cotizantes en la bolsa nacional de valores de la India (NSE), los autores comprobaron en su investigación que las empresas crearon valor para sus accionistas en términos de valor agregado económico (EVA) y valor agregado de mercado (MVA) durante los periodos comprendidos entre el año 2010 y el 2014. Ellos afirman que el EVA tiene un mejor poder predictivo en el análisis del desempeño financiero de una empresa que otros métodos tradicionales.

Para Stephens \& Bartunek (1997) EVA es una herramienta poderosa por varias razones: alinea el comportamiento de los empleados con la generación de valor de los accionistas. Beneficia la compensación de incentivos para empleados de la medición de rendimiento tradicional que compara los resultados reales presupuestados, y es relativamente fácil de comunicar y entender.

En la literatura se logra establecer que las fórmulas para determinar el EVA (1) y el MVA (2) son:

$$
\text { EVA }=\text { ROAI-WACC } \times \mathrm{Cl} \quad(1)
$$

Equivalente a:

$E V A=R O(1-T)-\left[\left(\% P^{*} K e\right)+\left(\% D^{*} K d^{*} 1-T\right)\right] \times C l$

Donde: 
Cuadro 1

Variables EVA

\begin{tabular}{|c|l|}
\hline $\mathrm{RO}=$ & Resultado operacional \\
\hline $\mathrm{ROAl}=$ & Resultado operacional después de impuesto \\
\hline $\mathrm{T}=$ & Tarifa de impuesto vigente para el año gravable \\
\hline $\mathrm{WACC}=$ & Costo Ponderado de Capital \\
\hline$\% \mathrm{P}=$ & Porcentaje de participación del Patrimonio en las fuentes de financiación \\
\hline $\mathrm{Ke}=$ & Costo del Patrimonio \\
\hline$\% \mathrm{D}=$ & Porcentaje de Patrimonio \\
\hline $\mathrm{Kd}=$ & Costo de deuda \\
\hline $\mathrm{Cl}=$ & Capital invertido por accionistas y acreedores \\
\hline
\end{tabular}

Fuente: Elaboración propia

MVA=Valor presente del EVA (2)

Equivalente a:

MVA=Valor de la empresa - Capital Invertido

Donde:

Cuadro 2

Variables MVA

\begin{tabular}{|c|l|}
\hline Valor de la empresa $=$ & Acciones expresadas en Cantidad * Precio cada acción \\
\hline Capital Invertido $=$ & Valor del Capital Invertido en estados financieros \\
\hline
\end{tabular}

Fuente: Elaboración propia

De estas fórmulas, se logra identificar que el EVA representa los ingresos residuales que quedan después de que se hayan reconocido todos los costos, incluido el costo de oportunidad del capital social empleado. Es decir, depende tanto de la eficiencia operativa como de la gestión del balance; en este caso la administración debe ser cuidadosa con la gestión de los elementos del estado de situación financiera, representado en activos, pasivos y patrimonio, a mayores activos y mayores capitales de financiación, resulta en costos de capital superiores a los necesarios. Para Garcia (2009) "el valor agregado se produce cuando la organización obtiene una rentabilidad superior a los costos incurridos en la financiación de la actividad económica, esta financiación entendida como los costos generados por los pasivos y el patrimonio" (p.40).

Por su parte investigadores como Griffith (2012) cuestiona la validez del valor económico agregado (EVA), pone en tela de juicio que este sea una medida para pronosticar rendimientos y plantea que en su origen y fundamentación es un sistema de compensación para trabajadores basado en el principio de maximización de la riqueza de los inversionistas.

\section{Metodología}

Esta investigación de tipo cuantitativo, pretendió determinar la existencia de relación entre el valor económico agregado, el valor agregado del mercado, y dos de los principales indicadores de resultados contables: rentabilidad neta y rentabilidad operativa, tomando como referencia los estados financieros (2015 a 2019) de uno de los grupos empresariales más importantes de Colombia. La investigación sustentada desde el paradigma positivista, formula hipótesis con la intención de comprobar la posible relación (correlación) ente los constructos mencionados en el cuerpo de éste trabajo. Además, se apoya en el estructuralismo, desde donde propone el modelo descrito gráficamente en el Gráfico 1. La técnica (de análisis de datos) utilizada para determinar la 
existencia o no, de relación entre los constructos es la correlación estadística, desde la cual se puede comprobar si ésta es, positiva o negativa.

\subsection{Hipótesis del modelo}

Teniendo en cuenta el modelo Machado \& Da Cuhna (2011) se plantean cuatro dimensiones de análisis. La variable resultados contables representada en los indicadores de desempeño denominados: resultados operacionales (RO) y los resultados netos (RN) del grupo económico en los periodos objeto de estudio, el valor agregado del mercado (MVA) y valor agregado del mercado (MVA), estas conforman el modelo empírico (Ver gráfico 1). A partir de lo anterior, se definen en total seis hipótesis a contrastar, estas son:

Hipótesis 1. El valor agregado del mercado (MVA) está fuertemente relacionado con el valor económico agregado (EVA) del grupo empresarial.

Hipótesis 2. El valor agregado del mercado (MVA) está fuertemente relacionado con los resultados operacionales del grupo empresarial.

Hipótesis 3. Los resultados netos del grupo empresarial están fuertemente relacionados con el resultado operacional.

Hipótesis 4. Los resultados netos del grupo empresarial están fuertemente relacionados con el valor económico agregado (EVA).

Hipótesis 5. El valor económico agregado (EVA) del grupo empresarial está fuertemente relacionado con los resultados operacionales.

Hipótesis 6. Los resultados netos del grupo empresarial están fuertemente relacionados con el valor agregado del mercado (MVA).

\section{Gráfico 1}

Esquema de hipótesis

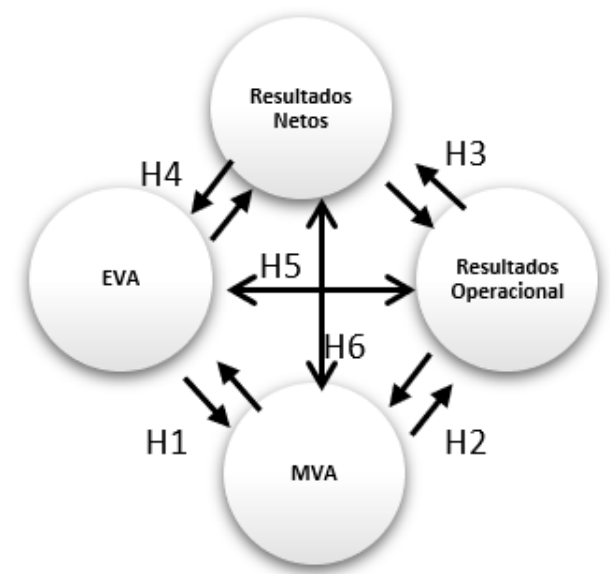

Fuente: Elaboración propia

Para comprobar la correlación de las hipótesis propuestas, se recurrió al análisis estadístico de regresión y correlación y se estimó el modelo de regresión lineal simple que se presenta a continuación: 
$\boldsymbol{Y}_{i, t}=\boldsymbol{\beta}_{o}+\boldsymbol{\beta}_{1} \boldsymbol{X}_{i, t}+\boldsymbol{\mu}_{j, t}$

Donde:

\begin{tabular}{|l|l|}
\hline $\boldsymbol{Y}_{\dot{j}, t}=$ & Variable dependiente relacionada con el grupo empresarial i, al final del periodo t \\
\hline $\boldsymbol{\beta}_{o}=$ & Efectos individuales específicos para cada unidad. \\
\hline $\boldsymbol{\beta}_{1}=$ & Coeficiente asociado a la variable explicativa \\
\hline $\boldsymbol{X}_{i_{t}}=$ & Variable independiente relacionada con el grupo empresarial i al final del año t \\
\hline $\boldsymbol{\mu}_{i_{t}}=$ & Perturbación aleatoria \\
\hline
\end{tabular}

Para el caso del análisis se tendrá en cuenta que una vez analizados los resultados pueden generarse los siguientes escenarios:

Formula del coeficiente de correlación:

$$
r=\frac{\sigma x y}{\sigma x \sigma y}
$$

Donde:

$$
\begin{aligned}
& \sigma x y=\text { Covarianza de la variable dependiente } \mathrm{Y} y \text { de la variable independiente } \mathrm{X} . \\
& \sigma x=\text { Covarianza de la variable independiente } \mathrm{X} \text {. } \\
& \sigma y=\text { Covarianza de la variable dependiente } \mathrm{Y} . \\
& \text { Resultados posibles de coeficiente de correlación: } \\
& r<0 \text { dependencia positiva (directa) } \\
& r>0 \text { dependencia negativa (inversa) } \\
& r=0 \text { no relación }
\end{aligned}
$$

\section{Resultados}

En el periodo de estudio se logró establecer que el grupo económico generó un aumento sostenido de sus utilidades operacionales, siendo el año 2016 el de mejor desempeño, dado que tuvo un aumento equivalente al $75 \%$ con respecto al año 2015 , esto genera una utilidad neta superior en un $38 \%$ mayor al 2015 . Sin embargo, es necesario señalar que debido a aparentes demoras en ejecuciones obras y a bajos índices de ingresos durante el año 2017 el grupo empresarial genero utilidades netas inferiores al 20\% con respecto al año 2015 (Ver Tabla 1).

Tabla 1

Utilidad del operacional y Neta del ejercicio

\begin{tabular}{lccccc}
\hline & $\mathbf{2 0 1 9}$ & $\mathbf{2 0 1 8}$ & $\mathbf{2 0 1 7}$ & $\mathbf{2 0 1 6}$ & $\mathbf{2 0 1 5}$ \\
\hline Utilidad Neta (RN) & $\$ 1.256 .137$ & $\$ 1.194 .118$ & $\$ 906.583$ & $\$ 1.132 .002$ & $\$ 648.319$ \\
Utilidad Operacionales (RO) & $\$ 4.533 .576$ & $\$ 4.083 .282$ & $\$ 4.254 .304$ & $\$ 4.121 .410$ & $\$ 2.976 .232$ \\
\hline
\end{tabular}

Fuente: Elaboración propia a partir de estados financieros consolidados

Con relación al indicador EVA se evidencia resultados negativos en todos los periodos, a pesar de generar sosteniblemente utilidades netas durante el periodo de estudio se ha generado destrucción de valor (ver tabla 2 ), esto se debe a que la estructura de costo del capital de financiación es superior a la rentabilidad generada en el capital de inversión. En este tipo de escenarios García (2003) recomienda "aumentar la utilidad operativa sin 
tener que aumentar la inversión, liberar fondos ociosos, invertir en proyectos con rentabilidad superior al costo del capital o desinvertir en actividades poco rentables" (p.153).

Tabla 2

Determinación del EVA y MVA en el grupo económico

\begin{tabular}{|c|c|c|c|c|c|c|}
\hline Concepto & Simbología & 2019 & 2018 & 2017 & 2016 & 2015 \\
\hline Costo del patrimonio & $\mathrm{Ke}$ & $3,90 \%$ & $4,70 \%$ & $4,43 \%$ & $4,59 \%$ & $4,58 \%$ \\
\hline Patrimonio & $\mathrm{P}$ & 26.490 .257 & 25.572 .737 & 24.307 .148 & 23.634 .596 & 22.974 .090 \\
\hline Deuda + Patrimonio & $D+P$ & 50.998 .454 & 48.647 .881 & 47.567 .948 & 44.749 .574 & 42.080 .401 \\
\hline Participación del patrimonio & $P /(D+P)$ & $51,94 \%$ & $52,57 \%$ & $51,10 \%$ & $52,82 \%$ & $54,60 \%$ \\
\hline Costo del patrimonio ponderado & Ke pond. & 0,020278146 & 0,024713505 & 0,022633728 & 0,024266951 & 0,025008863 \\
\hline Costo de la deuda & $\mathrm{Kd}$ & $4,95 \%$ & $4,61 \%$ & $4,64 \%$ & $4,60 \%$ & $3,81 \%$ \\
\hline Impuesto & $\mathrm{t}$ & $33 \%$ & $33 \%$ & $33 \%$ & $33 \%$ & $33 \%$ \\
\hline Costo de la deuda después de imp & $\mathrm{Kd}(1-\mathrm{t})$ & $3 \%$ & $3 \%$ & $3 \%$ & $3 \%$ & $3 \%$ \\
\hline Deuda & $\mathrm{D}$ & 24.508 .197 & 23.075 .144 & 23.260 .800 & 21.114 .978 & 19.106.311 \\
\hline Deuda + Patrimonio & $D+P$ & 50.998 .454 & 48.647 .881 & 47.567 .948 & 44.749 .574 & 42.080 .401 \\
\hline participación de la deuda & $D /(D+P)$ & $48,06 \%$ & $47,43 \%$ & $48,90 \%$ & $47,18 \%$ & $45,40 \%$ \\
\hline Costo de la deuda ponderado & Kd pond. & 0,015936512 & 0,014635779 & 0,015199036 & 0,014547029 & 0,011602592 \\
\hline Costo de capital & WACC & $3,621 \%$ & $3,935 \%$ & $3,783 \%$ & $3,881 \%$ & $3,661 \%$ \\
\hline EVA & & $-590.753,82$ & $-720.141,26$ & $-893.043,92$ & $-604.907,04$ & $-892.305,71$ \\
\hline MVA & & 16.833 .286 & 16.425 .891 & 16.046 .107 & 15.606 .828 & 14.929 .946 \\
\hline
\end{tabular}

Fuente: Elaboración propia a partir de estados financieros consolidados

\subsection{Test de hipótesis}

A partir de los resultados arrojados se logró confirmar las hipótesis 2 y 6 , es decir, la variable de rentabilidad neta (RN) con un $87,63 \%$ y la variable rentabilidad operativa (RO) con $84,31 \%$ tiene una correlación muy significativa frente al MVA, esto significa que las expectativas del mercado se encuentran más alineadas a las variables de rentabilidad y explican de mejor forma el comportamiento en comparación a la generación de EVA que tuvo una correlación débil del 54,01\% (Ver tabla 3).

Por su parte, la variable EVA tiene mayor correlación con la rentabilidad operativa (RO) en los años de estudio según el coeficiente de correlación en un $86,47 \%$, en segunda instancia la rentabilidad neta que tiene un $60,79 \%$, por último, el MVA con un nivel del 54,01\%, confirmando de esta forma las hipótesis 1, 4 y 5 . Por su parte, la rentabilidad neta $(\mathrm{RN})$ y la rentabilidad operativa, son altamente dependientes en su cálculo contable y según el modelo se encuentra estrechamente ligadas en un $85,07 \%$

Tabla 3

Coeficiente de Correlación de Pearson

\begin{tabular}{|c|c|c|c|c|}
\cline { 2 - 5 } \multicolumn{1}{c|}{} & MVA & EVA & RO & RN \\
\hline MVA & & 0,5401 & 0,8431 & 0,8763 \\
\hline EVA & 0,5401 & & 0,8647 & 0,6079 \\
\hline RO & 0,8431 & 0,8647 & & 0,8507 \\
\hline RN & 0,8763 & 0,6079 & 0,8507 & \\
\hline
\end{tabular}

Fuente: Elaboración propia

Con relación al coeficiente de determinación, el cual determina el porcentaje de variabilidad se logra establecer que la rentabilidad neta (RN) presenta los valores más significativos (superior al 70\%) con relación a las otras variables y la rentabilidad operacional (RO) es más significativa para las variables MVA y rentabilidad neta (RN) (ver tabla 4). Por su parte existe una relación débil entre el EVA y las variables MVA y RO. 
Tabla 4

\begin{tabular}{|c|c|c|c|c|}
\multicolumn{1}{c|}{ Coeficiente de determinación (R2) } \\
\cline { 2 - 5 } \multicolumn{1}{c|}{} & MVA & EVA & RO & RN \\
\hline MVA & & 0.2916 & 0.7108 & 0.7679 \\
\hline EVA & 0.2916 & & 0.3695 & 0.7477 \\
\hline RO & 0.7108 & 0.3695 & & 0.7236 \\
\hline RN & 0.7679 & 0.7477 & 0.7236 & \\
\hline
\end{tabular}

Fuente: Elaboración propia

\section{Conclusiones}

En la literatura ampliamente se plantea que el EVA y el MVA superan a los demás indicadores de desempeño financiero y en especial a los reportes contables, en lo referente a la generación de valor, se debe tener presente que este tipo de modelos están alineados a los principios del modelo económico neoliberal, este último impulsa la financiarización de las organizaciones.

A partir de esta investigación fue posible establecer que existe una relación significativa entre las medidas de desempeño contable representadas en la rentabilidad neta y la rentabilidad operativa y la variable valor agregado de mercado. Esto permite inferir que la información contable hasta el momento es una fuente de información fundamental para la determinación de valor en las organizaciones. En especial por los avances generados en la implementación de nuevos principios de reconocimiento, medición presentación y revelación basados en estándares de alta calidad, denominados Normas Internacionales de Información Financiera -NIIF.

Todavía hay controversia significativa sobre la validez de EVA y el MVA como la respuesta final a la necesidad de medir la generación de valor financiero. La literatura está llena de ejemplos y casos prácticos que apoyan mayoritariamente la utilización de estos métodos. Para resolver finalmente, la verdadera importancia del EVA y del MVA es necesario que se realicen más estudios empíricos a lo largo de series temporales más largas y con una muestra más representativa.

\section{Referencias}

Banerjee, A. (2000). Linkage between Economic Value Added and Market Value: An Analysis . Vikalpa, 25(3), 23-36. doi:10.1177/0256090920000304

Bhasin, M. L. (2013). Economic Value Added and Shareholders' Wealth Creation: Evidence from a Developing Country . International Journal of Finance and Accounting, 2(4), 185-198. doi:10.5923/j.ijfa.20130204.01

Brabazon, T., \& Sweeny, B. (1998). Economic value added-really adding something new ? Accountancy Ireland, $30,14-45$.

Chari, L. (2009). Measuring Value Enhancement Through Economic Value Added : Evidence from Literature. The IUP Journal of Applied Finance, 15(9), 46-62.

Eswara, M., \& Prasad, M. V. (2015). An Empirical Study on Economic Value-Added and Market Value-Added of Selected Indian FMCG Companies. SSRN Electronic Journal, 49-66. doi:10.2139/ssrn.2679755

Farzad, J., Degel, J., \& Degner, J. (2020). Economic Value Added (EVA) and Stock Returns. The Financier, 7(2), 115-118.

Garcia, O. (2009). Administración Financiera. Fundamentos y Aplicaciones (Cuarta ed.). Libre expresión creativos S.A.S.

García, S. O. (2003). Valoración de empresas, gerencia del valor y EVA. Medellín: Prensa Moderna Impresores. 
Griffith, J. (2012). The True Value of EVA . Journal of Allergy and Clinical Immunology, 130(2), 556. doi:10.1016/j.jaci.2012.05.050

Li Bonilla, F. (1969). El Valor Económico Agregado (Eva) en el Valor del Negocio. . Revista Nacional de Administración, 1(1), 55-70. doi:10.22458/rna.v1i1.284

Machado, C., \& Da Cuhna, T. (2011). Estudio y medición de la correlación entre el valor económico añadido y el valor de mercado agregado en un grupo empresarial cotizado en la bolsa NYSE Euronext. Cuadernos de Contabilidad, 12(31), 455-468.

Reddy, N. V., Rajesh, M., \& Reddy, T. N. (2011). Valuation through EVA and Traditional Measures an Empirical Study. International Journal of Trade, Economics and Finance, 19-23. doi:10.7763/ijtef.2011.v2.73

Stephens, K. R., \& Bartunek, R. R. (1997). What is economic value added? A practitioner's view. Business Credit, 99(4), 39-42.

Stewart, G. B. (1991). The quest for value: a guide for senior managers. New York: HarperBusiness.

Villegas, M. G. (2016). NIIF y MIPYMES: retos de la contabilidad para el contexto y la productividad. Cuadernos de Administración, 29(53), 49-76.

Wet, J., \& Hall, J. (2004). The relationship between EVA , MVA and leverage. 12(1), 39-59.

Zafiris, N., \& Bayldon, R. (1999). Economic value added and market value added A simple version and application. Accounting Research, 3(2), 83-105. Obtenido de http://repositorio.unan.edu.ni/2986/1/5624.pdf

Esta obra está bajo una Licencia Creative Commons Attribución-NoCommercial 4.0 International

\section{(c) BY-NC}

